\title{
Antimicrobial Peptide Brevinin-2isb, New Drug Candidates Enhance the Innate Immune Response and Cured Caenorhabditis Elegans With Methicillin-Resistant Staphylococcus Aureus (MRSA)
}

\author{
Hui Xie, Xueli Chen, Qi Zeng, Yonghua Zhan, Xinyi Xu, Dan Chen* and Jimin Liang* \\ School of Life Science and Technology, China
}

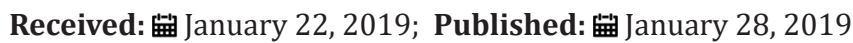

*Corresponding author: Dan Chen, School of Life Science and Technology, China

Jimin Liang, School of Life Science and Technology, China

\begin{abstract}
Antimicrobial peptide is supposed to solve the problem of drug resistance by pathogenic bacteria. Brevinin-2ISb is rich in amphibian skin and has been reported to have a wide range antimicrobial activity especially for MRSA bacteria (G+). The MRSA can also severely affect the behavioral indicators of nematodes. By treated with Brevinin-2ISb(MIC 4.35 $\pm 0.9 \mu \mathrm{M}$ ). The longevity of nematode and the survival rate of MRSA infection were significantly increased. C. elegans innate immune genes are induced expression, include hsf-1, irg-1, irg-2, C29F3.7, F49F1.6, K05D8.5, F56D6.2, F28D1.3, F38A1.5 ZK6.7, lys-1, spp-1, T20G5.7 and abf-1,by more than 5 to 25.5-fold. Brevinin-2ISb was shown to play an important rolein the enhancement of host immunity. The pmk-1, skn-1, and daf-2/daf-16 are essential in the Brevinin-2ISb induced by using C. elegans mutants. Compared with C. elegans and human in their conservation of innate immune pathways, the results of this study suggest that the peptide of Brevinin-2ISb not only has strong antibacterial activity, but may also play body immunity enhanced role in human.
\end{abstract}

Keywords: Brevinin-2ISb; Innate Immune Response; Caenorhabditis elegans; MRSA

\section{Introduction}

Antibacterial peptides(AMPs), which in organisms that defend the body against exogenous pathogen. AMPs are an important part of the body's immune system, and generally composed of 10 50 amino acid [1]. In recent years, drug resistance has become a research focus in the subject area. Traditional antibiotics such as penicillin are expected to change to new antibacterial peptide drugs [2]. Some AMPs are supposed to solve the problem of drug resistance by pathogenic bacteria [3]. Brevinin-2 is firstly separated from the Japanese bog frog skin [4]. By Dec 31st, 2017, there had been 86 peptides of Brevinin-2 family in Antimicrobial Peptide Database (APD) [5]. the primary structure of Brevinin-2 family has a big difference in its family members. From the Brevinin-2 peptides of the pool frog and belly frog, the gramnegative bacterium Escherichia coli bacteria has inhibitor activity (MIC $<10 \mathrm{mmol} / \mathrm{L})$, the gram-positive bacteria Staphylococcus aureus and Candida albicans fungus has inhibitory activity [68]. Compared to the Brevinin-1 family, the hemolysis activity of
Brevinin-2 family is weak. So Brevinin-2 family as an antibacterial drug candidate spotential [9]. The immune response of C. elegances mediated through the p38 mitogen-activated protein kinase (PMK$1)$,transforming growth factor $\beta$ (TGF- $\beta$ ), and the DAF-2/DAF16insulin-like and ZIP-2 pathways (17-20). Recently, antimicrobial peptide Brevinin-2ISb was demonstrated to protect $C$. elegans. In the present study, we tested the effects of on host immunity and MRSA pathogenicity using the $C$. elegans infection model with ATCC33591. We further examined the effect of Brevinin-2 family, especially for Brevinin-2ISb has the predominantonhost immunity. Moreover, we a number of mutant lines of $C$. elegans were utilized to determine the role of various signaling pathways in the Brevinin2ISb related immune response.

\section{Methods and Results}

\section{Determinate Motor Ability of $C$. Elegans}

To study the effect of MRSA on nematode movement, Stage L4 larvae were compared among the control group (OP50),the 
experimental group (MRSA), and the methicillin sensitive Staphylococcus aureus (MSSA) control group on NGM medium. We suggest that MRSA has multiple drug resistance characteristics similar to non-resistant Staphylococcus aureus, such as MSSA. After 3 min, the pharyngeal twitch, head swings, body bending, u turn, forward and backward movement were recorded as athletic ability index. The experiment was repeated 20 times every 30 seconds for statistical analysis. The $C$. elegans were extracted from NGM medium after $3 \mathrm{~min}$, and placed with OP50 on as and surface. The trajectory of the nematode was clearly visible as a sine curve. In the presence of MSSA, nematode movements were limited to a certain extent; the number of pharyngeal twitches, u turn, and forward movements were significantly decreased. In the presence of MRSA, the trajectory of $C$. elegans irregular sine curve peaks decreased. Motor ability was significantly reduced, with the exception of backward movement. Both kinds of $S$. aureushad an obvious effect on the movement abilities of $C$. elegans, especially MRSA (Figure 1). Each experiment was repeated three times.

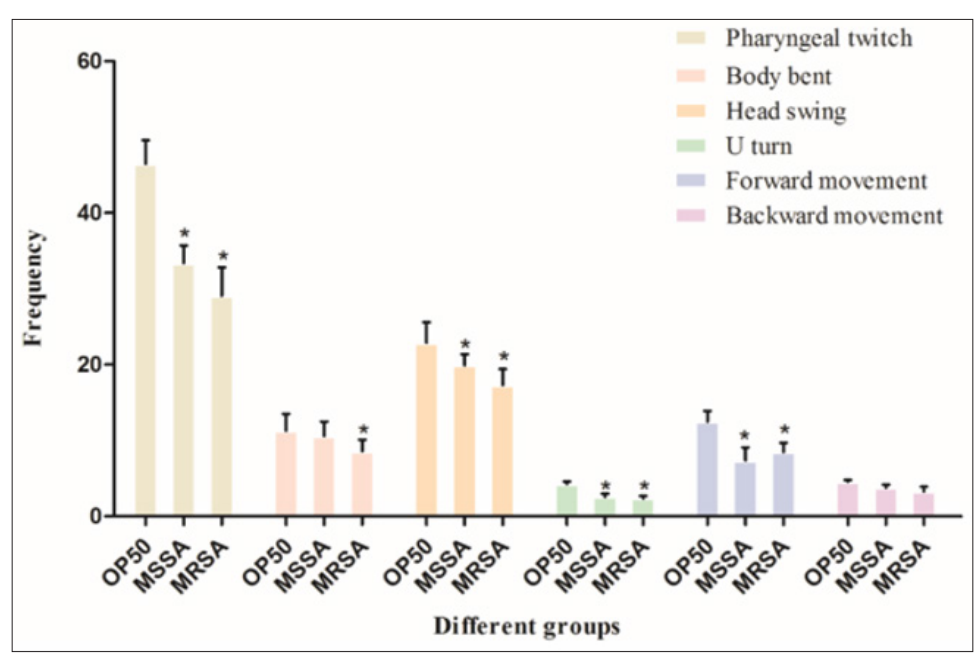

Note: From left to right: number of pharyngeal twitch, body bent, head swing, $u$ turn, forward, and backward movements. Compared with group OP50* means $\mathrm{p}<0.05$

Figure 1: Motor ability of C. elegans in different groups.

\section{Effects of Brevinin-2isb on the Longevity of Nematodes}

The longevity of nematode is a main basis for the study of aging process. The effects of minimum inhibitory concentration Brevinin$2 \mathrm{ISb}$ on the longevity of nematode were compared. Brevinin-2 ISb was dissolved in NGM plate and survival data were obtained at $12,24,36,48,60,72,84$ and 96 hours respectively. The survival rate of OP50 nematode in control group was compared. Survival curves of nematodes were plotted for the whole life cycle (Figure 2). The results showed that the minimum inhibitory concentration Brevinin-2ISb could make the survival curves of nematodes move up. Compared with the control group (OP50), the average and maximum longevity of nematodes in Brevinin-2ISb group were significantly prolonged, and the average longevity of nematodes in Brevinin-2ISb group was higher (more than 20\%) than that in the control group. Each experiment was repeated three times.

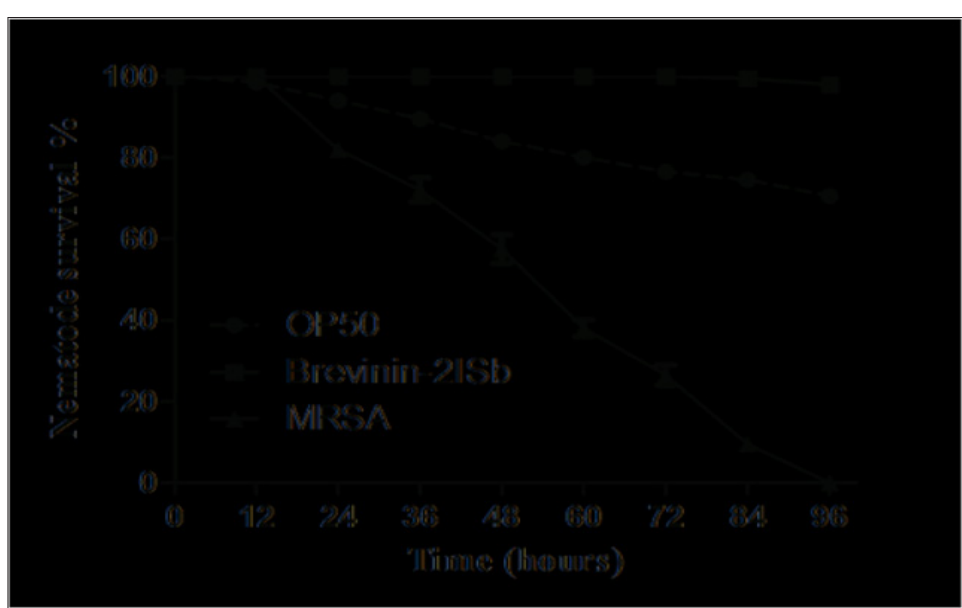

Figure 2: The survival of Caenorhabditis elegans (C.elegans) assessed. Staphylococcu saureus strain Brevinin-2ISb and Staphylococcus aureusstrain ATCC33591 (MRSA) were compared to Escherichia coli(OP50) as a control group. 


\section{Brevinin-2isb Protect C. Elegans from MRSA Infection}

To verify the anti-infective therapeutic potential of Brevinin2ISb, a screening in 12-well plates was performed with MRSA-C. elegans. Brevinin-2ISb were screened and demonstrated increased survival rates of MRSA-infected nematodes (Figure 3). Brevinin-
2ISb peptides contributed to a survival rate of more than $60 \%$ and increased the survival rate after $96 \mathrm{~h}$ compared to control group. The living worms were fed on E. coli OP50 during the assay. Brevinin-2ISb antimicrobial peptides identified above significantly affected nematode survival (Figure 4).

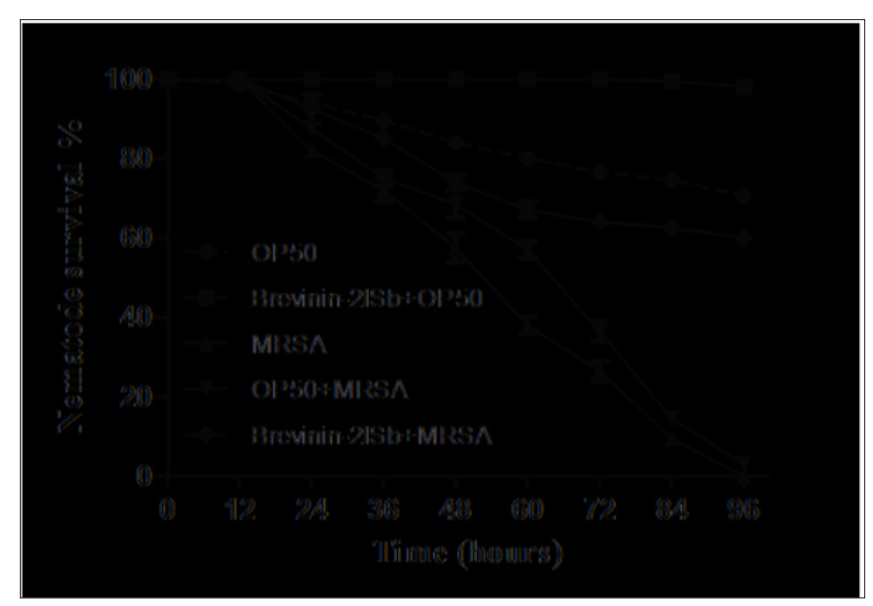

Figure 3: The survival of MRSA-infected C. elegansassessed by liquid-based assayusing Brevinin-2 family peptides.

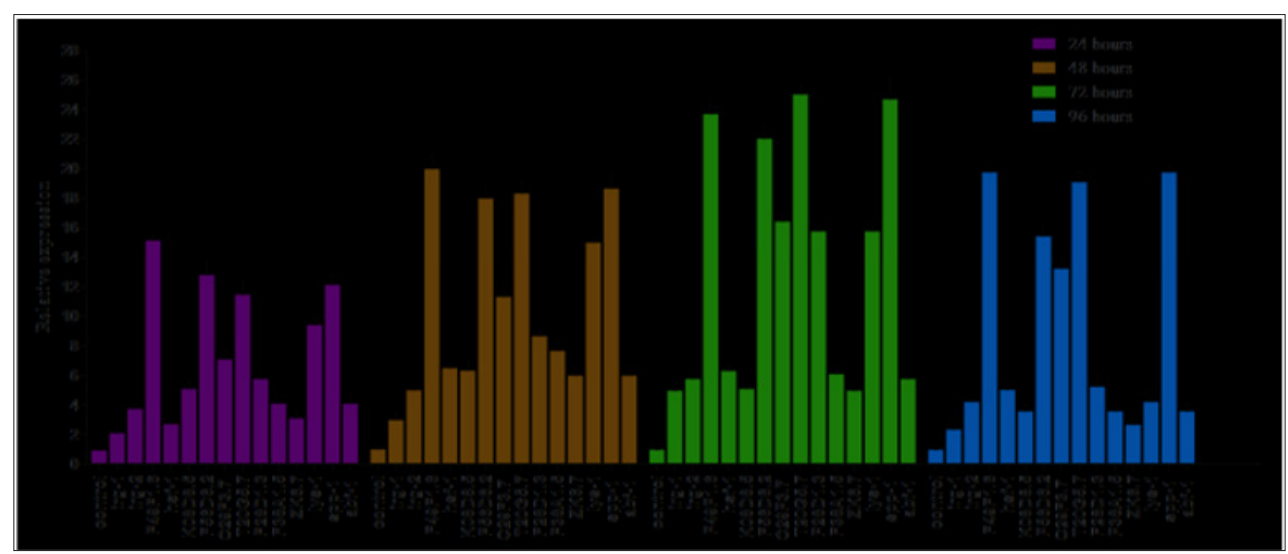

Figure 4: The relative gene expression of immune response genes in C. elegans after treatment with four Brevinin-2 family peptides. Compared with control group OP50, all treatment groups $\mathrm{p}<0.05$ or $\mathrm{p}<0.001$.

\section{Discussion}

In previous studies [10] , a four-day continuous determination of gene expression was then conducted in order to further study the effect of the peptides on MRSA inhibition and the effect of DAF-2/DAF-16 on the immune system of C. elegans. Brevinin-2OA3,Brevinin-2ISb, and Brevinin-2TSa significantly up-regulated both the mRNA and protein levels of the key antimicrobial gene lys7 in the DAF-2/DAF-16 pathway during 24-96 h. In this study ,we had firstly found in this work that Brevinin-2ISb was observed to extend the life span of infected and non-infected nematodes as well as enhance the expression of the tested immune response genes of adult worms, again suggesting an important role of Brevinin-2ISb in the positive modulation of immunity in the animals. C. elegans innate immune genes are induced expression by Brevinin-2ISb, include hsf-1, irg-1, irg-2, C29F3.7, F49F1.6, K05D8.5, F56D6.2, F28D1.3, F38A1.5 ZK6.7, lys-1, spp-1, T20G5.7 and abf-1,by more than 20 -fold at least. The pmk-1, daf-2/daf-16, and skn-1(these up-regulated genes belong to these innate immune pathways) are essential in the Brevinin-2ISb induced by using C. elegans mutants. Compared with C. elegans and human in their conservation of innate immune pathways, the results of this study suggest that the peptide of Brevinin-2ISb not only has strong antibacterial activity, but may also play body immunity enhanced role in human. The immune modulation effect of Brevinin-2ISb may have resulted from a variety of the bioactive compounds that it contains. The work presented here, using the model animal C. elegans, supports a beneficial effect of Brevinin-2ISb in host immune modulation. Further studies are required to elucidate the mechanism(s) of the immune modulation effect of Brevinin-2ISb.

\section{Funding}

This project was funded by the National Key R\&D Program of China (2018YFC0910600), Fundamental Research Funds for the 
Central Universities JB171207, and supported by the National Natural Science Foundation of China under Grant Nos. 81227901, 61471279, 81530058, and the Natural Science Basic Research Plan in Shaanxi Province of China under Grant No. 2015JZ019, and the Fundamental Research Funds for the Central Universities (NSIZ021402).

\section{References}

1. HK Liu, TM Hung, HC Huang, IJ Lee, CC Chang, et al. (2015) Bai-HuJia-Ren-Shen-Tang Decoction Reduces Fatty Liver by Activating AMPActivated Protein Kinase In Vitro and In Vivo. Evid Based Complement Alternat Med 2015: 651734.

2. K Yang, C Ma, M Zhou, L Wang, R Li, et al. (2016) Identification and bioactivity evaluation of a novel bradykinin inhibitory peptide from the skin secretion of Chinese large odorous frog, Odorrana livida. Journal of Peptide Science 22(3): 181-185.

3. Y Sun, Q Li, Z Li, Y Zhang, J Zhao, L Wang (2012) Molecular Cloning, Expression, Purification, and Functional Characterization of Palustrin2CE, an Antimicrobial Peptide of Rana chensinensis. Bioscience Biotechnology and Biochemistry 76(1): 157-162.

4. N Morikawa, K Hagiwara, T Nakajima (1992) Brevinin-1 and -2, unique antimicrobial peptides from the skin of the frog, Rana brevipoda porsa. Biochem Biophys Res Commun 189(1): 184-190.

\section{ISSN: 2574-1241}

DOI: 10.26717/BJSTR.2019.13.002446

Dan Chen, Jimin Liang. Biomed J Sci \& Tech Res

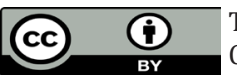

This work is licensed under Creative Commons Attribution 4.0 License

Submission Link: https://biomedres.us/submit-manuscript.php
5. GS Wang, X Li, Z Wang (2016) APD3: the antimicrobial peptide database as a tool for research and education. Nucleic Acids Res 44(D1): D1087-D1093.

6. JM Conlon, GJ Power, YH Abdel Wahab, PR Flatt, H Jiansheng, et al. (2008) A potent, non-toxic insulin-releasing peptide isolated from an extract of the skin of the Asian frog, Hylarana guntheri (Anura:Ranidae). Regul Pept 151(1-3): 153-159.

7. A Di Grazia, F Cappiello, A Imanishi, A Mastrofrancesco, M Picardo, et al. (2015) The Frog Skin-Derived Antimicrobial Peptide Esculentin1a(1-21)NH2 Promotes the Migration of Human HaCaT Keratinocytes in an EGF Receptor-Dependent Manner: A Novel Promoter of Human Skin Wound Healing?. PLoS One 10(6): e0128663.

8. B van der Meijden, JA Robinson (2015) Synthesis of a polymyxin derivative for photolabeling studies in the gram-negative bacterium Escherichia coli. Journal of Peptide Science 21(3): 231-235.

9. Zhao, Y Sun, Z Li, Q Su (2011) Molecular cloning of novel antimicrobial peptide genes from the skin of the Chinese brown frog, Rana chensinensis. Zoolog Sci 28(2): 112-117.

10. Hui Xie, Yonghua Zhan, Xueli Chen, Qi Zeng (2018) Brevinin-2 Drug Family-New Applied Peptide Candidates Against Methicillin-Resistant Staphylococcus aureus and Their Effects on Lys-7 Expression of Innate Immune Pathway DAF-2/DAF-16 in Caenorhabditis elegans. Dan Chen and Jimin Liang Appl Sci 8(12): 2627.

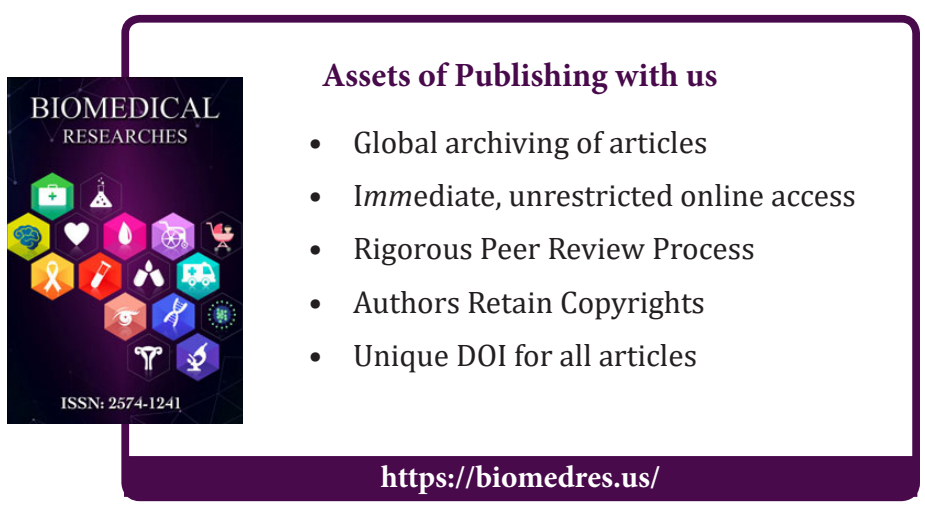

Cite this article: Hui Xie, Xueli Chen, Qi Zeng, Yonghua Zhan, Xinyi Xu, Dan Chen, Jimin Liang. Antimicrobial Peptide Brevinin-2isb, New Drug Candidates Enhance the Innate Immune Response and Cured Caenorhabditis Elegans With Methicillin-Resistant Staphylococcus Aureus (MRSA). 